\title{
Structural Study of Transition Metal Carbides
}

\author{
P. BHARDWAJ* \\ High Pressure Research Lab., Department of Physics, Barkatullah University \\ Bhopal-462026, India
}

(Received December 10, 2011; in final form May 3, 2012)

\begin{abstract}
In this research paper we have discovered the structural phase transition and elastic properties of transition metal carbides ( $\mathrm{TaC}$ and $\mathrm{HfC}$ ). Phase transition pressures are associated with a sudden collapse in volume showing the incidence of first order phase transition. At ambient condition the present compounds exhibit rock salt $(\mathrm{NaCl})$ structure, they transform into cesium chloride $(\mathrm{CsCl})$ structure under high pressure. The phase transition pressures and associated volume collapses obtained from present potential model show a generally good agreement with the available literature. The elastic constants and bulk modulus are also reported for the present compounds.
\end{abstract}

PACS: 62.20.de, 62.20.dq, 62.50.-p

\section{Introduction}

The carbides of the transition metals in groups IV-VI have extremely high melting points. In addition to their stability at high temperatures, these compounds are extremely hard finding industrial use in cutting tools and wear-resistant parts. Their hardness is retained to very high temperatures, and they have low chemical reactivity. Approximate preparation of the transition metal carbides is simple, but ensuring a given stoichiometry and purity against oxygen contamination is notably difficult. Group IV carbides are difficult to purify without melting. Slow diffusion of carbon in all of the refractory carbides results in stoichiometry gradients which are difficult to detect in bulk materials but which may compromise the material strength, hardness, and high-temperature behaviour. The tantalum carbide system is relatively easy to free of oxygen impurities, but due to the slow rate of carbon diffusion it tends to have inhomogeneities in its bulk composition. These interesting properties of the transition metal carbides have attracted attention of both theoretical and experimental workers [1-11]. At ambient temperature and pressure these transition metal carbides exhibit sixfold coordinated $\mathrm{NaCl}\left(B_{1}\right)$ structure. However under pressure they show a first order structural phase transition from $\mathrm{NaCl}\left(B_{1}\right)$ to $\mathrm{CsCl}\left(B_{2}\right)$ structure.

The electronic band structure and $\mathrm{X}$-ray photoelectron spectra of $\mathrm{TaC}$ and $\mathrm{HfC}$ has been performed by Ihara et al. [7]. The trends in elasticity and electronic structure of transition metal nitrides and carbides have been studied by $\mathrm{Wu}$ et al. [8]. Recently high pressure phase transi-

* e-mail: purveebhardwaj@gmail.com tion of carbides of Ta and Hf has been studied using the first principle calculations by Shrivastava et al. [9]. The computations have been performed using ground state total energy calculation approach of the system. The first principle calculations on the electronic structure of $\mathrm{TiC}_{x} \mathrm{~N}_{1-x}, \mathrm{Zr}_{x} \mathrm{Nb}_{1-x} \mathrm{C}$, and $\mathrm{HfC}_{x} \mathrm{~N}_{1-x}$ alloys have been performed by Zaoui et al. [10]. High pressure behaviour and structural properties of $\mathrm{HfC}$ and $\mathrm{TaC}$ have been predicted by Singh et al. [11] using an interionic potential theory. This interionic potential theory is not able to explain the Cauchy discrepancy in second order elastic constants.

Looking at the fact that these compounds are less explored, we applied our three-body interaction potential (TBIP) model to these compounds to study high pressure phase transition and other properties. The need of inclusion of three-body interaction forces was emphasized by many workers for the enhancement of results [12-14]. Earlier calculations for $B_{1}-B_{2}$ transitions were based on two-body potential mainly. They concluded that possible reasons for disagreements include the failure of the two-body potential model. These studies were based on two-body potentials and could not explain the Cauchy violations $\left(C_{12} \neq C_{44}\right)$. They remarked that results could be improved by including the effect of non-rigidity of ions in the model.

It is seen from the current literature that TBIP model used and developed by Singh and co-workers [15-18] has been found to be remarkably successful in giving the unified description of structural and elastic properties of ionic and semiconducting crystals [16-18]. In this TBIP model, the three-body interactions owe their origin to the quantum mechanical foundation and also to the phenomenological approach $[19,20]$ in terms of the transfer 
(or exchange) of charge between the overlapping electron shells of the adjacent ions in solids. The present approach has been extended to include the Hafemeister-Flygare (HF) type [21] overlap repulsion operative up to the second neighbour ions. Also, Tosi and co-workers [22] have demonstrated the significance of van der Waals (vdW) attraction due to the dipole-dipole $(\mathrm{d}-\mathrm{d})$ and dipolequadruple $(\mathrm{d}-\mathrm{q})$ interactions to describe the cohesion in ionic solids and they are generally ignored in the first principle calculations.

The purpose of this work is to investigate the structural and elastic properties of $\mathrm{TaC}$ and HfC. The rest of this paper is prepared as follows: the method of calculation is given in Sect. 2; the results and conclusion are presented and discussed in Sect. 3 .

\section{Potential model and method of calculation}

Application of pressure directly results in compression leading to the increased charge transfer (or three-body interaction effect [15]) due to the deformation of the overlapping electron shell of the adjacent ions (or non-rigidity of ions) in solids.

These effects have been incorporated in the Gibbs free energy $(G=U+P V-T S)$ as a function of pressure and three-body interactions (TBI) [15], which are the most dominant among the many-body interactions. Here, $U$ is the internal energy of the system equivalent to the lattice energy at temperature near zero and $S$ is the entropy. At temperature $T=0 \mathrm{~K}$ and pressure $(P)$ the Gibbs free energies for rock salt $\left(B_{1}\right.$, real $)$ and $\mathrm{CsCl}\left(B_{2}\right.$, hypothetical $)$ structures are given by

$$
\begin{aligned}
& G_{B 1}(r)=U_{B 1}(r)+P V_{B 1}(r), \\
& G_{B 2}\left(r^{\prime}\right)=U_{B 2}\left(r^{\prime}\right)+P V_{B 2}\left(r^{\prime}\right),
\end{aligned}
$$

with $V_{B 1}\left(=2.00 r^{3}\right)$ and $V_{B 2}\left(=1.54 r^{\prime 3}\right)$ as unit cell volumes for $B_{1}$ and $B_{2}$ phases, respectively. The first terms in (1) and (2) are lattice energies for $B_{1}$ and $B_{2}$ structures and they are expressed as:

$$
\begin{aligned}
& U_{B_{1}}(r)=\frac{-\alpha_{m} z^{2} e^{2}}{r}-\frac{\left.12 \alpha_{m} z e^{2} f_{(} r\right)}{r}-\left(\frac{C}{r^{6}}+\frac{D}{r^{8}}\right) \\
& \quad+6 b \beta_{i j} \exp \left(\left(r_{i}+r_{j}-r\right) / \rho\right) \\
& \quad+6 b \beta_{i i} \exp \left(\left(2 r_{i}-1.414 r\right) / \rho\right) \\
& \quad+6 b \beta_{j j} \exp \left(\left(2 r_{j}-1.414 r\right) / \rho\right), \\
& \quad+8 b \beta_{i j} \exp \left(\left(r_{i}+r_{j}-r^{\prime}\right) / \rho\right) \\
& \quad+3 b \beta_{i i} \exp \left(\left(2 r_{i}-1.154 r^{\prime}\right) / \rho\right) \\
& \quad+3 b \beta_{j j} \exp \left(\left(2 r_{j}-1.154 r^{\prime}\right) / \rho\right),
\end{aligned}
$$

with $\alpha_{m}$ and $\alpha_{m}^{\prime}$ as the Madelung constants for $\mathrm{NaCl}$ and $\mathrm{CsCl}$ structure, respectively. $C\left(C^{\prime}\right)$ and $D\left(D^{\prime}\right)$ are the overall van der Waals coefficients of $B_{1}\left(B_{2}\right)$ phases, $\beta_{i j}(i, j=1,2)$ are the Pauling coefficients. $Z e$ is the ionic charge and $b(\rho)$ are the hardness (range) parameters, $r\left(r^{\prime}\right)$ are the nearest neighbour separations for $\mathrm{NaCl}$ $(\mathrm{CsCl})$ structure, $f(r)$ is the three-body force parameter.

These lattice energies consist of long range Coulomb energy (first term), three-body interactions corresponding to the nearest neighbour separation $r\left(r^{\prime}\right)$ (second term), vdW interaction (third term), energy due to the overlap repulsion represented by $\mathrm{HF}$ type potential and extended up to the second neighbour ions (remaining terms).

\section{Results and discussion}

The Gibbs free energies contain three model parameters $[b, \rho, f(r)]$. The values of these parameters have been evaluated using the first and second order space derivatives of the cohesive energy $(U)$ expressed as

$$
\begin{aligned}
& \left(\frac{\mathrm{d} U}{\mathrm{~d} r}\right)_{r=r_{0}}=0, \\
& \left(\frac{\mathrm{d}^{2} U}{\mathrm{~d} r^{2}}\right)_{r=r_{0}}=9 k r_{0} B_{\mathrm{T}},
\end{aligned}
$$

and following method adopted earlier [23-25]. Using these model parameters and the minimization technique, phase transition pressures of transition metal carbides have been computed. The input data of the crystal and calculated model parameters are listed in Table I. The $B_{1}(\mathrm{NaCl})$ structure is most stable in these compounds and at high pressure they transform to body centre $B_{2}$ $(\mathrm{CsCl})$ structure. As the stable phase is associated with minimum free energy of the crystal, we have followed the technique of minimization of the Gibbs free energies of real and hypothetical phases. We have minimized $G_{B 1}(r)$ and $G_{B 2}\left(r^{\prime}\right)$ given by Eqs. (3) and (4) at different pressures in order to obtain the interionic separations $r$ and $r^{\prime}$ corresponding to $B_{1}$ and $B_{2}$ phases associated with minimum energies.

TABLE I

Input parameters and generated model parameters for transition metal carbides.

\begin{tabular}{c|c|c|c|c|c}
\hline \hline \multirow{2}{*}{ Solid } & \multicolumn{2}{|c|}{ Input parameters } & \multicolumn{3}{c}{ Model parameters } \\
\cline { 2 - 6 } & $r_{0}[\AA]$ & $B[\mathrm{GPa}]$ & $b\left[10^{-12} \mathrm{erg}\right]$ & $\rho[\AA]$ & $f(r)$ \\
\hline $\mathrm{TaC}$ & $2.235^{a}$ & $324^{a}$ & 2.14623 & 0.346 & -0.13052 \\
$\mathrm{HfC}$ & $2.325^{a}$ & $238^{a}$ & 1.37913 & 0.319 & -0.14837 \\
\hline
\end{tabular}

${ }^{a}$ Ref. [1]

The factor $\Delta G\left[\mathrm{G}_{B 1}(r) \sim G_{B 2}\left(r^{\prime}\right)\right]$ plays an important role in stability of structures. The phase transition occurs when $\Delta G$ approaches zero $(\Delta G \rightarrow 0)$. The phase transition pressure $\left(P_{\mathrm{t}}\right)$ is the pressure at which $\Delta G$ approaches zero. At $P_{\mathrm{t}}$ these compounds undergo a $B_{1}-B_{2}$ transition associated with a sudden collapse in volume. Figure 1 shows our presently computed phase transition pressure for NaCl-type $\left(B_{1}\right)$ to $\mathrm{CsCl-type}\left(B_{2}\right)$ structures 
in $\mathrm{TaC}$ at 93.2 $\mathrm{GPa}$ and in $\mathrm{HfC}$ at $86.4 \mathrm{GPa}$, respectively. The present phase transition pressures illustrated by arrows in Figs. 1 and 2 and their values have been listed in Table II and compared with available results. It is interesting to note from Table II and Figs. 1 and 2 that the phase transition pressures $\left(P_{\mathrm{t}}\right)$, obtained from our model, are in general in closer agreement with available results $[9,11]$

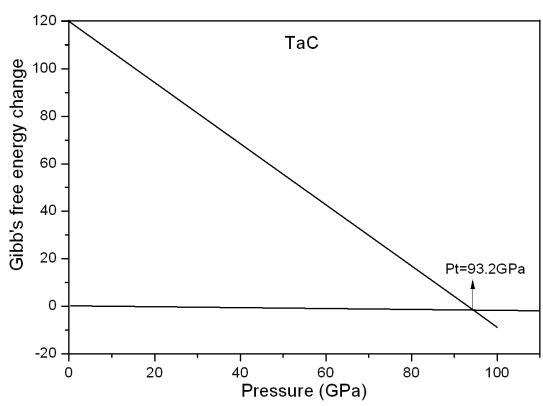

Fig. 1. Variation of Gibbs free energy with pressure for $\mathrm{TaC}$.

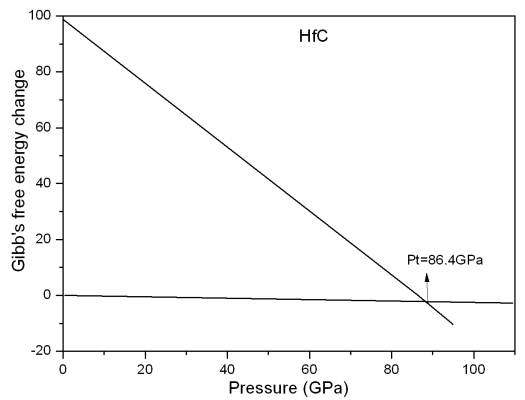

Fig. 2. Variation of Gibbs free energy with pressure for $\mathrm{HfC}$.

TABLE II

Phase transition and volume change of transition metal carbides.

\begin{tabular}{c|c|c|c|c}
\hline \hline \multirow{2}{*}{ Solid } & \multicolumn{2}{|c|}{$\begin{array}{c}\text { Phase transition pressure } \\
{[\mathrm{GPa}]}\end{array}$} & \multicolumn{2}{c}{ Volume collapse } \\
\cline { 2 - 5 } & Present & Others & Present & Others \\
\hline $\mathrm{TaC}$ & 93.2 & $96^{a}, 641^{b}$ & 6.73 & $7.6^{a}$ \\
$\mathrm{HfC}$ & 86.4 & $85^{a}, 485^{b}$ & 5.14 & $6.8^{a}$ \\
\hline
\end{tabular}

${ }^{a}$ Ref. [9], ${ }^{b}$ Ref. [11]

During the phase transition from $\mathrm{NaCl}$ to $\mathrm{CsCl}$, the volume discontinuity in pressure volume phase diagram identifies the occurrence of first order phase transition. The compression curves are plotted in Figs. 3 and 4 . The values of the volume collapses $(-\Delta V(P) / V(0))$ are depicted in Table II and compared with corresponding theoretical results $[9,11]$. The experimental values of volume collapses are not available for the present compounds.

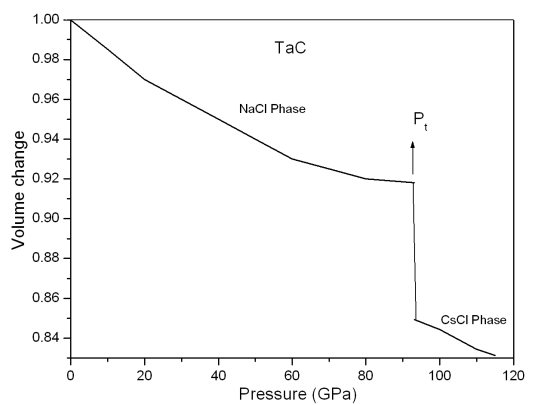

Fig. 3. Variation of volume change $V_{P} / V_{0}$ with pressure for $\mathrm{TaC}$.

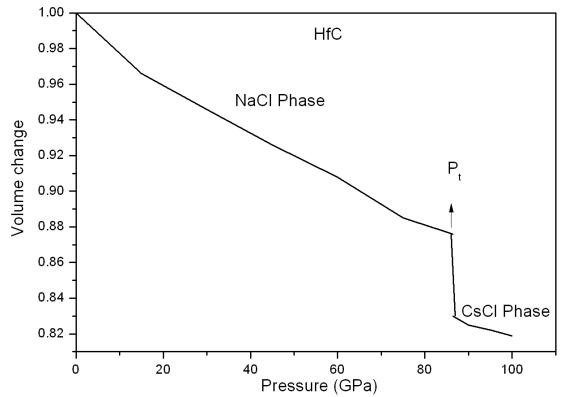

Fig. 4. Variation of volume change $V_{P} / V_{0}$ with pressure for HfC.

We have calculated the second order elastic constants of the materials under study. Also, we could reproduce the correct sign of the elastic constants $\left(C_{11}-C_{12}\right)$. The study of second order elastic constants (SOECs) under pressure is important as $C_{11}$ represents elasticity in length and $C_{12}$ and $C_{44}$ are shape related elastic constants. The SOECs and pressure derivatives of transition metal carbides are given in Table III. Our present values of SOECs are close to available theoretical data [11]. The calculated SOECs and their pressure derivatives could not be compared due to lack of experimental data on them but the values of SOECs are of the same order as reported by others [11].

TABLE III

Calculated values of elastic constants (in GPa), bulk modulus (in GPa), pressure derivative and Cauchy discrepancy of transition metal carbides.

\begin{tabular}{c|c|c|c|c|c|c}
\hline \hline Solid & $C_{11}$ & $C_{12}$ & $C_{44}$ & $B$ & $\mathrm{~d} B / \mathrm{d} P$ & $\delta$ \\
\hline $\begin{array}{c}\mathrm{TaC} \\
\text { present } \\
\text { others }\end{array}$ & 6.29 & 1.64 & 1.53 & 3.19 & 0.326 & -0.14 \\
\hline $\begin{array}{c}\text { HfC } \\
\text { present } \\
\text { others }\end{array}$ & 4.651 & $0.74^{a}$ & $1.27^{a}$ & $3.18^{a}$ & - & - \\
\hline
\end{tabular}

${ }^{a}$ Ref. [11] 


\section{Conclusions}

In view of the overall achievements, it may be concluded that there is generally a good agreement of TBIP model with the available experimental and theoretical values.

Finally, it may be concluded that the present model has successfully predicted the compression curves and phase diagrams giving the phase transition pressures, associated volume collapses and elastic properties correctly for these transition metal carbides.

\section{References}

[1] L.E. Toth, Transition Metal Carbides and Nitrides, Academic Press, New York 1971.

[2] T. Amriou, B. Bouhafs, H. Aourag, B. Khelifa, S. Bresson, C. Mathieu, Physica B 325, 46 (2003).

[3] W. Weber, Phys. Rev. B 8, 5082 (1973).

[4] C.K. Jun, P.T.B. Shaffer, J. Less-Common Met. 4, 323 (1971).

[5] V.V. Nemoshkalenkov, P. Krivitskiai, P. Nesenjuk, L.I. Nikolajev, A.P. Shpak, J. Phys. Chem. Solids 36, 277 (1972).

[6] A.C. Lawson, D.P. Butt, J.W. Richardson, Ju Li, Philos. Mag. 87, 2507 (2007).

[7] H. Ihara, M. Hirabayashiand, H. Nakagawa, Phys. Rev. B 14, 1707 (1976).

[8] Z. Wu, X.-J. Chen, V.V. Struzhkin, R.E. Cohen, Phys. Rev. B 71, 214103 (2005).

[9] A. Shrivastava, Mamta Chauhana, R.K. Singh, Phase Transit. 84, 58 (2011).
[10] A. Zaoui, B. Bouhafs, P. Ruterana, Mater. Chem. Phys. 91, 108 (2005).

[11] A. Singh, M. Anayas, S.P. Sanyal, Phase Transit. 82, 576 (2009).

[12] C.E. Sims, G.D. Barrera, N. L Allan, Phys. Rev. B 57, 11164 (1998).

[13] B.S. Rao, S.P. Sanyal, Phys. Status Solidi B 165, 369 (1991).

[14] S. Froyen, M.L Cohen, J. Phys. C 19, 2623 (1983).

[15] R.K. Singh, Phys. Rep. 85, 259 (1982).

[16] R.K. Singh, S. Singh, Phys. Rev. B 39, 671 (1989); Phase Transit. 15, 127 (1997).

[17] R.K Singh, S. Singh, Phys. Rev. B 45, 1019 (1992).

[18] S. Singh. R.K. Singh, R. Rai, B.P. Singh, J. Phys Soc. Jpn. 68, 1269 (1999).

[19] P.O. Lowdin, Adv. Phys. 75, 1 (1932); J. Chem. Phys. 18, 113 (1961)

[20] S.O. Lundqvist, Ark. Fys. (Swedon) 12, 365 (1958).

[21] D.W. Hafemeister, H.F. Flygare, J. Chem. Phys. 43, 795 (1965).

[22] M.P. Tosi, F.G. Fumi, J. Phys. Chem. Solids 23, 359 (1962); M.P. Tosi Solid State Phys. 16, 1 (1964).

[23] P. Bhardwaj, S. Singh, N.K. Gaur, Centr. Eur. J. Phys. 6, 223 (2008).

[24] P. Bhardwaj, S. Singh, N.K. Gaur, Mat. Res. Bull. 44, 1366 (2009).

[25] P. Bhardwaj, S. Singh, Mat. Chem. Phys. 125, 440 (2011). 